\title{
MODELING OF ABRASIVE WATER JET MACHINING PROCESS
}

\author{
Aswathy K and Govindan P \\ Department of Physics, Payyanur College, Payyanur, \\ Assistant Professor, Department of Mechanical Engineering, Government College of \\ Engineering, Kannur,India
}

\begin{abstract}
:
This paper presents the modeling of abrasive water jet cutting and development of analytical expressions for material removal rate during abrasive water jet machining process. Water jet machining employs the principle of increasing the pressure of water and to subsequently discharge the water through an orifice. Normally, the diameter of the orifice varies from 0.8 to $1 \mathrm{~m}$. The water jet impinging on the chosen work surface generates sufficient force to cut the work material.Considering theoptimization of the process, few factors appearsto be influential.They are: velocity of the water jet, diameter of the nozzle and the average pressure of the jet.Furthermore, in the modeling, the explanation for higher width of kerf at the top than that of bottom is obtained.In this regard, the flow rate equation of water is determined. By using the expression for flow rate, the power equation for water jetis developed. With regard to material removal rate, a linear relationship with power of the water jet is evident. Additionally, the characteristics of kerf formed during cutting based on energy loss during cutting process using the relation between material removal rate and power of the water jetis assessed.
\end{abstract}

\section{KEYWORDS:}

Machining, water jet, modeling, kerf width, power and material removal rate.

\section{INTRODUCTION}

There are three major areas classified under advanced machining processes. These streams are mechanical, thermo-electrical and electro-chemical. One of such important mechanical advanced machining process is Abrasive Water Jet (AWJ) machining [1]. The abrasive water jet machining process do not generate any heat because of which there are not heat affected zones and residual stresses during the surface generation process. The inherent characteristic of the process is that it is non-contact and dynamic cutting process. The other notable benefits includethin kerf width, negligible heat affected zone, and flexibility during material removal [2]. Various classes of abrasives are usually applied during AWJ machining like garnet, olivine, aluminum oxide $\left(\mathrm{Al}_{2} \mathrm{O}_{3}\right)$, silica-sand, glass bead, silicon carbide(SiC), zirconium, etc [3, 4]. There are several advantages of abrasive water jet machining process such as no thermal alterations; flexibility in machining, larger applicability besides very small cutting forces. There are several distinguished advantages of AWJ technique. It is less sensitive to material properties and hence does not cause chatter, has no thermal effects, imposes minimal stresses on the work piece, and has high machining versatility and flexibility [5]. It has been always challenging to incorporate constricted tolerances on the components and parts machined using the process. In addition to the materials having superior properties, the machining quality can also be extended for the applications 
involving tight tolerances. This is in contrast to the machining techniques employed traditionally, wherein the hardness of the tool material is always higher than that of the work material [6]. The unconventional machining methods are contactless processes, which utilize the different sources and forms of energy for material removal. There are several unconventional machining processes commonly employed in industries. Abrasive water jet machining is one among the popular processes such as chemical machining, laser beam machining, ultrasonic machining, electrochemical machining and electrical discharge machining [7]. Being a potential eco-friendly machining technology, flexibility, and range to process different materials, abrasive water jet technology has become important for several recent industrial applications. Abrasive water jet machining process can be used to cut materials irrespective of reflectivity, specimen thickness, hardness and thermal conductivity [8]. The ability of the water jet machining process to cut materials of varying thickness has generated significant interest among the researchers. Based on the application situations, the cut-off thickness can be typically for stainless steel as $100 \mathrm{~mm}$, for aluminium as $120 \mathrm{~mm}$, for stone as $140 \mathrm{~mm}$ and $100 \mathrm{~mm}$ for glass. It is possible to generate complex features such as fine tabs and filigree structure and to machine contours of random orientations. Abrasive water jet machining completely eliminates thermal stresses and presence of slag. Furthermore, the tolerance level is tight as upto $0.1 \mathrm{~mm}$ on a material, eliminating the need for other process sequences [9].

In this paper, the material removal phenomenon in abrasive water jet machining is analyzed and modeled, considering the physical behavior of the process. The parameters governing the process are water pressure, nozzle diameter and jet velocity. The flow of water jet with the abrasives is mathematically modeled and analyzed. Furthermore, the material removal in the process is expressed as a function of known water jet parameters. The generation of kerf during water jet machining is correlated with the dynamics of water jets. The energy interactions during water jet is modeledat various processing conditions. Finally, a correlation between energy, kerf width and depth of cut is developed for the abrasive water jet machining process.

\section{BACKGROUND AND METHODOLOGY}

Knowing the velocity and mass distribution profiles would enable us to calculate the energy profile of the abrasive waterjet, which, beside the workpiece material properties, is the most important input parameter to modeling the material removal process. The energy distribution depends on the velocity profiles and mass distribution of each phase across the abrasive waterjet. Most of the kinetic energy is transported by the high velocity abrasive particles and the waterjet. Even if the air flow reaches supersonic velocities, this phase of the abrasive waterjet transports just a small amount of the total kinetic energy due to the low density of air. In water jet cutting, the kinetic energy of the water is high enough to remove the workpiece material, but in order to machine harder materials; a higher amount of kinetic energy has to be available. This energy can be stored in abrasive particles due to their higher density comparing to the water density. The available energy in the time interval $t_{j}$, during which an abrasive particle is applied such that the energy is dispersed, which is defined by eq. (1) and to the abrasive particles having the energy in kinetic form, defined by eq. (2),

$$
E_{K W J, j}=\frac{m_{W J, j} v_{W J, j}^{2}}{2}
$$




$$
E_{K A P, J}=E_{K A P, J}^{T}+E_{K A P, J}^{R}=\frac{m_{A P, J} v_{A P, J}^{2}}{2}+\frac{J_{A P, J} \cdot \omega_{A P, J}^{2}}{2}
$$

As evident from eq. (2), the kinetic energy of abrasive particles is composed by the energy stored in particle translation and energy stored in particle rotation. Inthe equations (1) and (2), $m_{w j, j}$ is the mass of the part of $\mathrm{WJ}$ associated to the time interval $t_{j}, v_{w j, j}$ the average velocity of the $\mathrm{WJ}$, while $v_{A P, J}$ is the velocity, $\omega_{A P, J}$ the rotational velocity and $J_{A P, J}$ the inertial momentum of the observed abrasive particle. By knowing the amount of the kinetic energy, the abrasive water jet is not fully defined. An important attribute for the workpiece material removal process is the distribution of energy across the abrasive water jet as well. In this way, the abrasive water jet would be fully defined quantitatively and qualitatively.

\subsection{Modeling of water jet-abrasive flow in water jet machining process}

The modeling of the abrasive water jet machining process is based on few important principles of fluid dynamics and fluid systems. They areBernoulli's principle (Fig. 1), intensifier and mixing chamber. In fluid dynamics, Bernoulli's principle states that for an inviscid, an increase in the speed of the fluid occurs simultaneously with a decrease in pressure or a decrease in the fluid's potential energy. The fundamental principle of the law of conservation of energy is used for an idealfluid and is as follows:

$$
\mathrm{P}+\frac{\rho_{w V_{w}}}{2}+\rho_{w} \mathrm{gh}=\mathrm{const}
$$

where, $\mathrm{p}$ - water pressure, $V_{w^{-}}$velocity of water, $\rho_{w^{-}}$density of water, $\mathrm{g}$ - acceleration due to gravity andh - height of the observed points above the reference plane. By observing the leakage of high pressure water jets in the air and using equation (3), one can determine the leakage velocity of water jet from a nozzle based on water pressure.

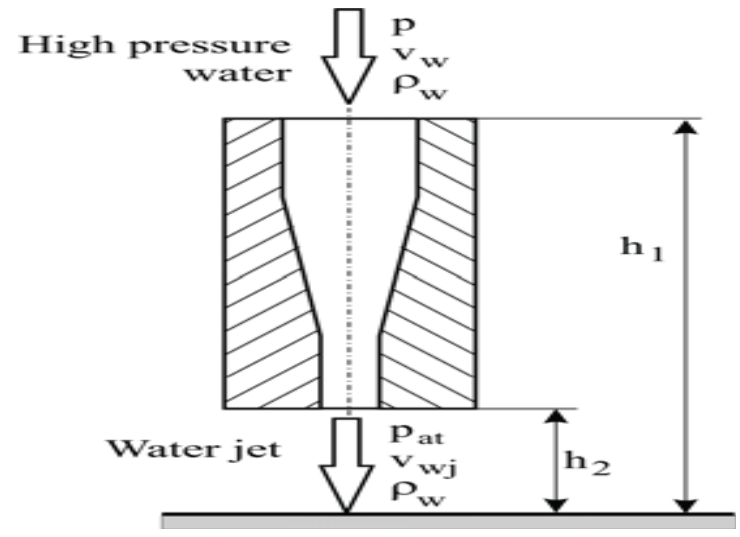

Fig. 1Bernoulli'stheorem applied to water jet machining process

$$
\mathrm{P}+\frac{\rho_{w} V_{w}^{2}}{2}+\rho_{w} \mathrm{~g} h_{1}=P_{a t}+\frac{\rho_{w} V_{w j}^{2}}{2}+\rho_{w} \mathrm{~g} h_{2} \text { or }
$$




$$
\begin{aligned}
& \mathrm{P}-P_{a t}=\frac{1}{2} \rho_{w}\left(V_{w j}^{2}-V_{w}^{2}\right)+\rho_{w} \mathrm{~g}\left(h_{1-} h_{2}\right) \\
& \text { For } P_{a t}<<\mathrm{P} ; V_{w j}>>V_{w} ; h_{1} \approx h_{2} \\
& \mathrm{P}=\frac{1}{2} \rho_{w} V_{w j}^{2}
\end{aligned}
$$

Now, few assumptions are made to simplify the expression into a generalized form. The assumptions are as follows:

- The height difference is fully neglected

- It is assumed that the speed of the water on nozzle entrance is negligible compared to the speed of the jet at the nozzle exit

- the atmospheric pressure (1 bar) is much smaller than the water pressure at the entrance to the nozzle (4000 bar)

- Based on these assumptions, the equation for calculating the velocity of the water jet after exiting the water nozzleis obtained as,

$$
V_{w j}=\sqrt{\frac{2 P}{\rho_{W}}}
$$

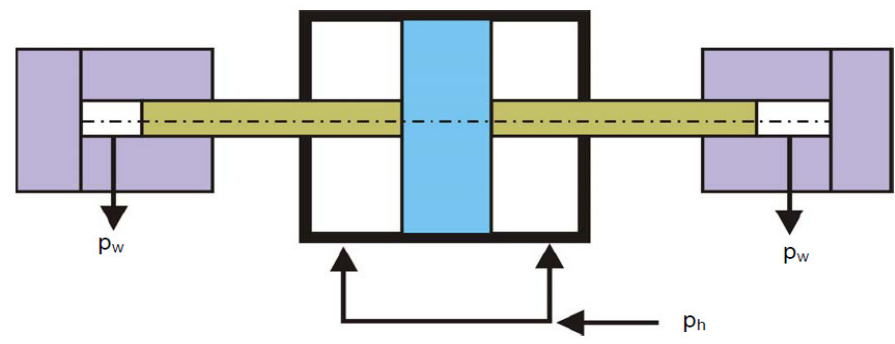

Fig. 2 Principle of hydraulic intensifier applied to water jet machining process

The mechanism of waterjet machining can also be assumed to be influenced by the principle of hydraulic intensifier (Fig. 2). In this, the hydraulic oil is supplied to the intensifier at pressure $P_{h}$ by thehydraulic power pack. Further, in the intensifier, the ratio of cross-sectional areas of the two cylinders is identified. Hence, the area ratio is estimated as, A ratio (A = A large / A small). Therefore, a pressure amplification is expected. This amplification happens at the small cylinder and is,

$$
\begin{aligned}
& P_{h} \times \mathrm{A}_{\text {large }}=P_{w} \times \mathrm{A}_{\text {small }} \\
& \mathrm{P}_{\mathrm{w}}=P_{h} \times \mathrm{A}_{\text {large }} / \mathrm{A}_{\text {small }} \\
& \mathrm{P}_{\mathrm{w}}=P_{h} \times \mathrm{A}_{\text {ratio }}
\end{aligned}
$$


In water jet machining, using direction control valve, the hydraulic unit drives the intensifier. Further, the water can be directly provided to the small cylinder of the intensifier. It can also be delivered through a pump known as booster pump. The pump then usually raises the pressure of the water to approximately 11 bar before delivering it to the intensifier. Thus, as the intensifier works, it delivers high pressure water. As the larger piston changes direction within the intensifier, there would be a drop in the delivery pressure. Cutting head consists of orifice, mixing chamber and focussing tube or insert where water jet is formed and mixed with abrasive particles to form abrasive water jet.Typical diameter of the flexible stainless steel pipes is of $6 \mathrm{~mm}$.

The velocity of the water jet thus formed can be estimated, assuming no losses as $v_{w j}=\sqrt{\frac{2 P}{\rho_{W}}}$ using Bernoulli's equation where, $\mathrm{p}$ is the water pressure and $\rho_{w}$ is the density of water. The orifices are typically made of sapphire. In commercial machines, the life of the sapphire orifice is typically around $100-150$ hours. In water jet machining, this high velocity water jet is used for the required application where as in abrasive water jet machining it is directed into the mixing chamber. The mixing chamber (Fig. 3) has a typical dimension of inner diameter $6 \mathrm{~mm}$ and a length of $10 \mathrm{~mm}$. In this case, the velocity of the water is very high and is issued from the orifice into the mixing chamber. As a result, a low pressure (vacuum) is created within the mixing chamber. By exploiting this principle and making use of the advantage, the metered abrasive particles are introduced into the mixing chamber through a port.

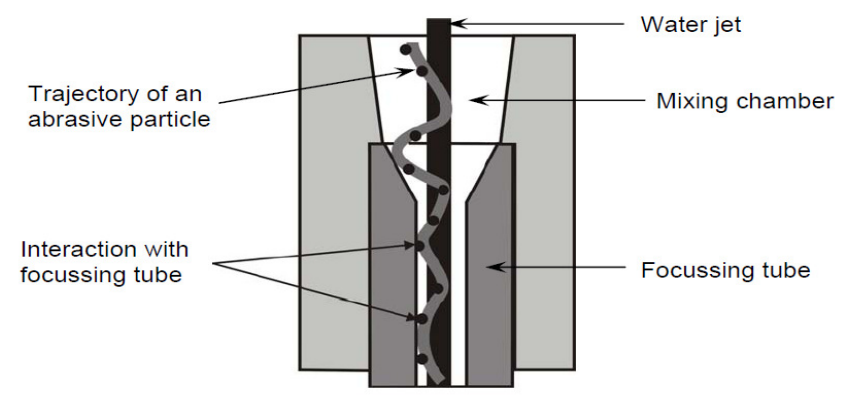

Fig. 3 A schematic diagram of the mixing process

The abrasive particles are introduced within the water jet. Finally,because of this mixing action, the abrasive water jet comes out of the focusing tube as fully developed. It si evident that during the process of mixing, the abrasive particles are gradually accelerated. This is because of the transfer of momentum from the water phase to abrasive phase. Further, the jet lastly leaves the focusing tube, phases, water and abrasive. At this moment, all are expected to be at same velocity. The mixing chamber, as shown in Fig. 3, is immediately followed by the focusing tube or the inserts. Abrasive particles during mixing try to enter the jet, but they are reflected away due to interplay of buoyancy and drag force. They go on interacting with the jet and the inner walls of the mixing tube, until they are accelerated using the momentum of the water jet.Considering the energy loss through water jet development at the orifice, the water jet velocity is estimated and obtained as given by the expression:

$$
v_{w j}=\psi \sqrt{\frac{2 P_{w}}{\rho_{w}}}
$$


where,

$\Psi=$ Orifice parameter known as the coefficient of velocity. The volume flow rate of water may be expressed as,

$$
\dot{q_{w}}=\varnothing \times V_{w j} \times A_{\text {orifice }}
$$

where, $A_{\text {orifice }}$ is the area of the orifice, given by,

$$
A_{\text {orifice }}=\frac{\pi}{4} d_{0}^{2}
$$

where $d_{0}$ is water orifice diameter,

$$
\begin{aligned}
& \dot{q}_{w}=\varnothing \times V_{w j} \times \frac{\pi}{4} d_{0}^{2} \\
& \dot{q}_{w=\varnothing \times \frac{\pi}{4}} d_{0}^{2} \times \psi \sqrt{\frac{2 P_{w}}{\rho_{w}}} \\
& \dot{q}_{w=c_{d}} \times \frac{\pi}{4} d_{0}^{2} \times \sqrt{\frac{2 P_{w}}{\rho w}}
\end{aligned}
$$

where,

$c_{d}=$ Coefficient of discharge of the orifice

Thus, it is essential to find out the total power of the jet of issuing waterjet. An expression for the same may be obtained as given in the following set of equations.

$$
\begin{aligned}
& P_{w j}=P_{w} \times q_{w} \\
& P_{w j}=p_{w} \times c_{d} \times \frac{\pi}{4} d_{0}^{2} \times \sqrt{\frac{2 p_{w}}{\rho_{w}}} \\
& P_{w j}=c_{d \times \frac{\pi}{4}} d_{0}^{2} \times \sqrt{\frac{2 p_{w^{3}}}{\rho_{w}}}
\end{aligned}
$$

A verylow pressure is generated within the mixing chamber as the high velocity water jet flows through orifice into the mixing chamber.At the same time, abrasive particles are allowed to move into the mixing chamber by means of a port (Fig. 4). The physical interactions of the water particles and the abrasive particles in the mixing chamber is highly complex to evaluate. Furthermore, during mixing process, the abrasive particles are progressively accelerated. This is because of the reason that there is transfer of momentum from the water phase to abrasive phase. The jet finally leaves the abrasive nozzle, phases, water and abrasive at a uniform velocity. 


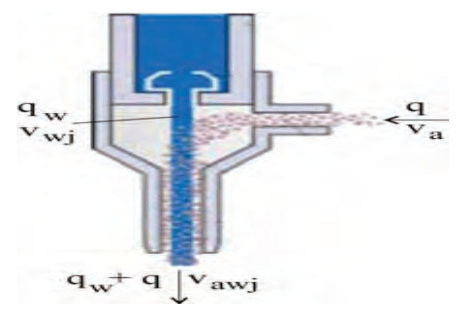

Fig. 4 Mixing process

where, q - abrasive particle flow rate, $V_{a}$ - initial velocity of abrasive particles.It is fully evident that both momentum and energy are not preserved due to losses that may occur during the mixing process. However, initially it would be assumed that no losses take place in momentum. With regard to this, the momentum of the jet before and after mixing is conserved. The relevant expressions are presented.

$$
\begin{gathered}
\sum(\dot{m v})_{\text {before }}=\sum(\dot{m v})_{a f t e r} \\
\left(m_{\text {air }} v_{\text {air }}+m_{w} v_{w j}+m_{a b r} v_{a b r}\right)_{b e f o r e}=\left(m_{a i r} v_{a i r}+m_{w} v_{w j}+m_{a b r} v_{a b r}\right)_{a f t e r}
\end{gathered}
$$

The total momentum of any closed system, i.e., the vector sum of the momentum vectors of all the elements in the system, is a constant. This principle is known as law of conservation of momentum. Therefore, it is evident that the momentum of air before and after the mixing process. The momentum variations can be neglected because of very low density of air. After the mixing process, both water and abrasive particlesreach the same velocity of abrasive water jet, and uniformity is maintained. In this case, the abrasive particles are applied into the water jet through the port of the mixing chamber. An evaluation shows that their velocity is also very low and their momentum can be neglected, for this state.

$$
\begin{aligned}
& \dot{m}_{w} v_{w j}=\left(\dot{m}_{w}+\dot{m}_{a b r}\right) v_{w j} \\
& v_{a w j}=\frac{\dot{m}_{w}}{\left(\dot{m}_{w}+\dot{m}_{a b r}\right)} v_{w j} \\
& v_{a w j}=\frac{1}{(1+R)} v_{w j}
\end{aligned}
$$

where,

$$
\mathrm{R}=\text { loading factor }=\frac{\dot{m}_{a b r}}{\dot{m}_{w}}
$$

The loss of momentum occurs as the abrasives strike the water jet, during the mixing process. The impingement occurs on the inner wall of the focusing tube several times, after which it is allowed to entrain. The velocity of abrasive water jet is, therefore, estimatedas,

$$
v_{a w j}=\eta \frac{1}{(1+R)} v_{w j}
$$


where, $\eta=$ momentum loss factor. The value of the momentum loss factor ranges from 0.65 to 0.85 . The abrasive flow rate determines the number of abrasive particles in contact, and at the same time, their kinetic energies. Hence, the energy of the abrasive particles can be expressed as:

$$
\begin{gathered}
E_{a w j}=\frac{1}{2} \mathrm{q} V_{w j}=\frac{1}{2} \mathrm{q} \eta^{2} \frac{v_{w j}^{2}}{\left[1+\frac{m_{a b r}}{m_{w}}\right]^{2}} \\
E_{a w j}=\frac{1}{2} \mathrm{q} V_{w j}=\frac{1}{2} \mathrm{q} \eta^{2} \frac{v_{w j} m_{w}^{2}}{\left[m_{w}+m_{a b r}\right]^{2}} \\
\text { But } \quad v_{w j}^{2}=\frac{2 P}{\rho_{v}}, \text { therefore, } \\
E_{a w j}=\frac{1}{2} \mathrm{q} \eta^{2} \frac{2 P}{\rho_{v}} \frac{m_{w}^{2}}{\left[m_{w}+m_{a b r}\right]^{2}}
\end{gathered}
$$

Otherwise it can be written as,

$$
E_{a w j}=\frac{1}{2} \mathrm{q} \eta^{2} \frac{2 P}{\rho_{v}} \frac{q_{w j}^{2}}{\left[q_{w j}+q\right]^{2}}
$$

\subsection{Modeling of material removal in abrasive water jet machining process}

In abrasive water jet machining process, the relationship between jet velocity, abrasive velocity, fluid pressure and the material removal is very important. The jet of water employed for the machining process can be considered as either pure water jet or abrasive water jet.In water jet machining process, mostly, continuous pure water jets in air issued from a nozzle having a circular cross section are used. The typical applications in industries involve cleaning, surface preparation, and cutting of soft materials.As comparted to pure water jets, the material removal capability is much higher. When an abrasive water jet is employed, the abrasive particles are accelerated in a stream of pure water. From the fundamental principles of water jets, and knowing that water jet helps accelerate the abrasive particles for material removal, the pressure of water jet is the governing factor. Therefore, it can be mathematically expressed as,

$$
\begin{aligned}
& \operatorname{MRR} \propto p_{w j} \\
& \operatorname{MRR} \propto c_{d} \times \frac{\pi}{4} d_{0}^{2} \times \sqrt{\frac{2 p_{w^{3}}}{\rho_{w}}} \\
& \operatorname{MRR}=u \times c_{d} \times \frac{\pi}{4} d_{0}^{2} \times \sqrt{\frac{2 p_{w^{3}}}{\rho_{w}}}
\end{aligned}
$$

The specific energy requirement is represented as $u$ and is therefore, considered as a property of the work material.

$$
P_{a b r}=\frac{1}{2} \dot{m}_{a b r}+v_{a w j}^{2}
$$


International Journal of Recent advances in Mechanical Engineering (IJMECH) Vol.4, No.3, August 2015

$$
\begin{aligned}
& P_{a b r}=\frac{1}{2} \dot{m}_{a b r}\left\{\eta \frac{1}{(1+R)} v_{w j}\right\}^{2} \\
& P_{a b r}=\frac{1}{2} \dot{m}_{w} \mathrm{R}\left\{\eta \frac{1}{(1+R)} v_{w j}\right\}^{2} \\
& P_{a b r}=\frac{1}{2} c_{d} \times \frac{\pi}{4} \times d_{0}^{2} \rho_{w} v_{w j} R \eta^{2}\left(\frac{1}{1+R}\right)^{2} v_{w j}{ }^{2} \\
& P_{a b r}=c_{d} \times \frac{\pi}{8} d_{0}^{2} \rho_{w} R \eta^{2}\left(\frac{1}{1+R}\right)^{2} v_{w j}{ }^{3} \\
& P_{a b r}=c_{d} \times \frac{\pi}{8} d_{0}^{2} \rho_{w} R \eta^{2}\left(\frac{1}{1+R}\right)^{2}\left(\frac{2 P_{w}}{\rho_{w}}\right)^{\frac{3}{2}} \\
& P_{a b r}=c_{d} \times \frac{\sqrt{2} \pi}{4} d_{0}^{2} \rho_{w} R \eta^{2}\left(\frac{1}{1+R}\right)^{2} \frac{P^{\frac{3}{2}}}{\rho_{w}^{\frac{1}{2}}} \\
& P_{a b r}=c_{d} \times \frac{\pi}{4} d_{0}^{2} R\left(\frac{\eta}{1+R}\right)^{2} P_{w}^{\frac{3}{2}} \sqrt{\frac{2}{\rho_{w}}}
\end{aligned}
$$

Thus it may be assumed that the material removal rate is proportional to the power of abrasive phase of AWJ. The water phase does not contribute to material removal in abrasive water jet machining.

$$
\operatorname{MRR}=\dot{Q}=\frac{p_{a b r}}{u_{j i b}}
$$

where,

$u_{j i b}=$ specific energy requirement in machining a material in abrasive water jet machining

Now ,

$$
\mathrm{MRR}=h_{t} \mathrm{w} V_{f}
$$

Where,

$h_{t}=$ depth of penetration

$$
\mathrm{w}=\text { width of the } \operatorname{kerf}=\left(w_{\text {top }}+w_{\text {bottom }}\right) / 2
$$

$\approx d_{i}$, the diameter of the focussing tube or nozzle or the insert

$V_{f}=$ traverse speed of the abrasive water jet or cutting speed

Generally,

$$
\operatorname{MRR}=\xi \frac{p_{a b r}}{u_{j i b}}
$$


where, $\xi$ is a coefficient, which considers several of other factors like sharpness or dullness of the abrasive, friability of the abrasives, stand-off distance, process inhomogenities etc. Therefore,

$$
h_{t}=\xi c_{d} \times \frac{\pi}{4} d_{0}^{2} R\left(\frac{\eta}{1+R}\right)^{2} \sqrt{\frac{2}{\rho_{w}}} \frac{p_{w^{\frac{3}{2}}}}{u_{j o b} d_{i v_{f}}}
$$

Now the machiningapproach should be selected in such a way so that maximization of $h_{t}$ takes place.

$$
\mathrm{R}=\frac{\dot{m}_{a b r}}{m_{w}}
$$

Optimal loading ratio is obtained and analyzed by applying differentiation with respect to the loading ratio, $\mathrm{R}$

$$
h_{t}=\mathrm{K} \frac{R^{2}}{(1+R)^{2}}
$$

Where, $\mathrm{K}$ is the constant.

$$
\begin{aligned}
& \frac{\partial h_{t}}{\partial R}=K(1+R)^{2}-2 R-2(1+R)^{2}=0 \\
& (1+R-2 R=0) \\
& 1-\mathrm{R}=0 \Rightarrow \mathrm{R}=1
\end{aligned}
$$

Thus theoretically maximum depth of penetration occurs at $\mathrm{R}=1$.

Phenomenon of generation of kerf during material removal: The generation of kerf is an important phenomenon in abrasive water jet machining and is to be modeled analytically. Fig. 5 show the cut produced by an AWJM in different sections, known as a kerf.The top of the kerf is wider than the bottom of the kerf. Generally, the top width of the kerf is equal to the diameter of the AWJ. Once again, diameter of the AWJ is equal to the diameter of the focusing tube or the insert if the standoff distance is around 1 to $5 \mathrm{~mm}$. The taper angle of the kerf can be condensed by increasing the cutting ability of the AWJ by analyzing various process conditions.

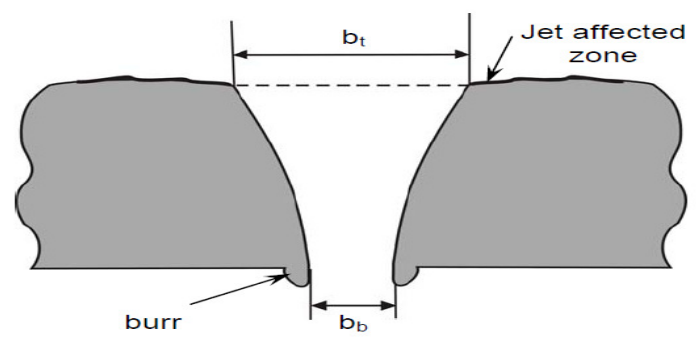

Fig. 5Cut generated by an AWJM in different sections

The generation of kerf during material removing is due to the energy loss during material removing. During the cutting process a certain amount of AWJ energy is absorbed by the 
material, which must be considered in the development of cutting models and in the abrasive water jet cutting process interpretation. It is known that afraction of the input energy generated during abrasive water jet machining is expended by the workpiece and contributes to the material destruction.As shown by Momber et al. about 1.0 per cent of the AWJ energy is used to remove the material. The rest of the energy may be dissipated by different mechanisms, such as friction and heat generation. An important part of the AWJ energy is occupied by the mixture of abrasive, water and wear particles after it leaves the workpiece.

\subsection{Modeling of energy interactions in abrasive water jet machining}

The energy which is absorbed during the cutting process, $E_{A B}$,can be estimated using,

$$
E_{A B}=E_{I N}-E_{L}
$$

where, $E_{I N}$ is the input energy of the abrasive water jet and $E_{L}$ is the amount of energy in the jet when it leaves the work piece. Many investigations in jet cutting have shown that a critical minimum energy $E_{C}$ (critical pump pressure, critical traverse rate) is necessary to destroy a material and to initiate the cutting process.

A critical situation will be found at the bottom of the cut in a workpiece $\left(h=h_{\max }\right)$. when the abrasive water jet does not have enough energy to penetrate the material, This simple assumption leads to,

$$
E_{A B\left(h=h_{\max }\right)}=E_{I N}-E_{L}
$$

Another critical condition exists in the case $h=0$. Here no energy is absorbed by the material and the total amount of the abrasive water jet energy goes into the exit jet. Thissituation leads to,

$$
E_{A B(h=0)}=0
$$

By introducing a parameter $\chi(\mathrm{h})$ which depends on the depth of cut and using equation (52), the situation can be described by equation (55) for all cases between these extremum:

$$
E_{A B}(h)=\chi(h)\left(E_{I N}-E_{L}\right) \quad 0<\mathrm{h}<h_{\max }
$$

For the conditions in equation (53), $\chi\left(\mathrm{h}=h_{\max }\right): 1$ is obtained, whereas equation (55) leads to the case $\chi(\mathrm{h}: \mathrm{O})=0$, thus the parameter $\chi(h)$ considers the energy absorption of the material during cutting. An energy loss during cutting process implies that there is a power loss occurs in the material removal of cutting process.

We know that, material removal rate is proportional to power of the water jet. Hence, as the time elapses, the material removal rate reduces along with power by an amount $\chi(h)$.We saw that at the surface of the material $\chi(h)$ is zero, that is $\chi(\mathrm{h}: \mathrm{O})=0$, which means that there is no energy absorption takes place by the material. 
But when depth of cut increases $\chi(h)$ increases and it will become $\chi\left(\mathrm{h}=h_{\max }\right): 1$. So the total amount of energy reduces. As a result the quantity of material removed at $\mathrm{h}=h_{\text {max }}$ will be less than that of at the surface, where $\mathrm{h}=0$.

Mathematically we can explain this as,

$\operatorname{MRR} \propto p_{w j}$

However,

$\mathrm{MRR}=h_{t} \mathrm{w} V_{f}$

where,

$h_{t}=$ depth of penetration

$\mathrm{w}=\left(w_{\text {top }}+w_{\text {bottom }}\right) / 2$ is the width of the kerf

$V_{f}=$ traverse speed of the AWJ or cutting speed

If $V_{f}$ is a constant, then,

$\mathrm{MRR} \propto h_{t} \mathrm{~W}$

When $h_{t}$ increases power reduced, hence MRR reduced because of equation (56). So in order to balance the equation (58), 'w' wil be reduced. That means when depth of penetration increases width of kerf decreases. So the kerf width at the top of the material is wider than the bottom.

\section{CONCLUSIONS}

Abrasive water jet machining is an efficient method for cutting materials. The flow of water jet and its interaction with abrasive particles is analytically modeled and analyzed. Based on this theoretical work, the following conclusions can be drawn:

- The modeling of abrasive water jet cutting has indicated that the calculated material removal rate is proportional to the power of the water jet, and at the same time, the area of the orifice. Thus, from the modeling studies, it is evident that the material removal rate (MRR)varies in proportion to the square of the diameter of the orifice.

- It is evident from the analytical calculations that during the process of material removal, there may occur some energy loss. Therefore, the kerf formed has a wider top width than that of bottom width.

- The material removal ratevaries in proportion to two important abrasive water jet factors. They are:i) power of the water jet and ii) energy of the water jet. Hence, an energy loss during cutting may cause a reduction in the material removal rate.

- It is evident that the energy loss is proportional to the removal of material. Because of this, if there is anenergy loss, there is a corresponding reduction in the material removal rate. The width of kerf should be decreased as the depth of penetration increases, which is again verified by the energy decrement. Therefore, the kerf is wider at the top than that the bottom. 


\section{ACKNOWLEDGEMENTS}

The authors would like to express their gratitude to Prof.Vinod Kumar T,Assistant professor at PayyannurCollege, for his leadership and the head of the Physics department Prof. K. C. Padmaja, for giving her support to this project. The authors also wish to express sincere thanks to Mr.Sreekesh.K,M.Tech student in Mechanical Engineering at Government College of Engineering, Kannur.

\section{REFERENCES}

[1] Hashish, M. (1993), Prediction models for AWJ machining operations, 7th American Water jet Conference, Seattle, Washington, pp. 205-216.

[2] Patrick, H. and Eu-Gene, N. (2012), Multi-modeling of abrasive water jet machining, PhD Thesis, Mc Master University, Canada.

[3] Wang, J. (2009), Particle velocity models for ultra-high pressure abrasive waterjets, Journal of Materials Processing Technology, v209, n9, pp. 4573-4577.

[4] EITObgy, M., Ng, E. G., Elbestavi, M. A. (2005), Modelling of abrasive waterjet machining: A New Approach, CIRP Annals-Manufacturing Technology, v54, n1, pp. 285-288.

[5] Hashish, M. (1984), A Modeling Study of Metal Cutting With Abrasive Waterjets, Journal of Engineering Materials and Technology, v106, n1, pp.88-100.

[6] EITObgy, M. (2007), Modeling of Abrasive Waterjet Machining Using a Multi-physics Approach, Mcmaster University, Canada.

[7] Momber, A. and Kovacevic, R. (1988), Principles of abrasive water jet machining, Springer.

[8] Jain, V. K. (2011), Advanced machining processes, Alllied publishers, New Delhi.

[9] Brandt, C. and Louis, H. (1998), Influence of the nozzlegeometry on abrasive water jets, Proc. of the 5th Int. Conf. on Water Jet Tech. New Delhi, India, 330-344. 